\title{
OPEN GABARAP ameliorates IL-1 $\beta$-induced inflammatory responses and osteogenic differentiation in bone marrow-derived stromal cells by activating autophagy
}

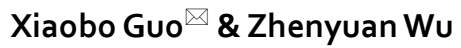

Bone mesenchymal stem cells (BMSCs) are the most commonly investigated progenitor cells in bone defect repair and osteoarthritis subchondral bone regeneration; however, these studies are limited by complex inflammatory conditions. In this study, we investigated whether pro-autophagic $\gamma$-aminobutyric acid receptor-associated protein (GABARAP) promotes BMSCs proliferation and osteogenic differentiation by modulating autophagy in the presence or absence of interleukin-1 beta (IL-1 $\beta$ ) in vitro. The expression levels of all relevant factors were evaluated by qRT-PCR or western blotting where appropriate. BMSCs differentiation were assessed by Alizarin Red, alkaline phosphatase, safranin O, and Oil Red O staining. Furthermore, the interactions between autophagy and osteogenic differentiation were investigated by co-treatment with the autophagy inhibitor 3-methyladenine (3-MA). As the results, we found that treatment with recombinant human His6GABARAP protein promoted cell proliferation, inhibited apoptosis, and reduced ROS generation by increasing autophagic activity, particularly when co-cultured with IL-1 $\beta$. Moreover, His6GABARAP could effectively increase the osteogenic differentiation of BMSCs. The expression levels of inflammatory factors were significantly decreased by His6-GABARAP treatment, whereas its protective effects were attenuated by 3-MA. This study demonstrates that GABARAP maintains BMSCs survival and strengthens their osteogenic differentiation in an inflammatory environment by upregulating mediators of the autophagy pathway.

Mesenchymal stem cells (MSCs), including BMSCs, display a robust proliferative capacity and multipotency that allows them to differentiate into adipocytes ${ }^{1}$, chondrocytes ${ }^{2}$, osteoblasts ${ }^{3}$, and other non-mesodermal lineage cells ${ }^{4}$ in different microenvironments. Therefore, these cells are considered promising therapeutic candidates for tissue engineering and display potential for clinical application in conditions such as osteoarthritis (OA). Previous studies have strongly indicated that BMSCs can effectively promote cartilage erosion regeneration in OA by not only differentiating into cartilage-forming chondrocytes but also modulating the behavior of subchondral bone ${ }^{5}$. However, the abnormal microenvironment of OA characterized by stress conditions, such as inflammation ${ }^{6}$ and oxidative stress ${ }^{7,8}$, reduces the survival capacity of BMSCs. Moreover, high pro-inflammatory cytokine levels inhibit BMSCs osteogenesis ${ }^{9}$. Therefore, approaches to promote BMSCs osteogenic differentiation and improve their cell survival under severe stress is particularly important for tissue renewal and subsequent regeneration ${ }^{10}$.

IL- $1 \beta$ is a primary initiator of inflammatory progression in $\mathrm{OA}^{11}$, with studies reporting that elevated IL- $1 \beta$ levels play a central role in inflammation-induced bone destruction ${ }^{12,13}$. Indeed, IL-1 $\beta$ expression levels are significantly higher in OA than in healthy sites and is associated with subchondral destruction ${ }^{14}$. Mechanistically, the inhibitory effects of IL- $1 \beta$ have been associated with MSCs proliferation and the activation of osteoclastogenesis ${ }^{15,16}$. IL-1 $\beta$ treatment can also dramatically induce the production of ROS, nitric oxide (NO), and proteolytic enzymes such as matrix metalloproteinase (MMP) during bone formation, thus inhibits BMSCs 
osteogenic differentiation and bone tissue generation in vitro and in vivo ${ }^{17-19}$. Although anti-inflammation has been studied for decades, our understanding of the mechanism underlying the effects of IL-1 $\beta$ on cell viability and osteoblast differentiation remains poor.

Previous studies have shown that the maintenance of bone mass stability is associated with the activation of cellular autophagy ${ }^{20}$, which is a highly conserved catabolic process that maintains cellular homeostasis and recycles degraded cytoplasmic materials ${ }^{21-23}$. Autophagy can also maintain MSCs stemness and protect cells against stress pathology signals, including ROS, inflammation, and metabolic precursors ${ }^{24,25}$; for instance, inhibiting autophagy in osteoblast-like cells increases oxidative stress and stimulates their apoptosis ${ }^{26}$. During osteogenesis, autophagy is critical for decreasing osteopenia by accelerating mineralization and osteogenesis in vitro and in vivo ${ }^{20,27}$, indicating that increased autophagy may maintain the BMSCs phenotype after IL- $1 \beta$ treatment and enable the promotion of osteoblast differentiation.

GABARAP is a member of the autophagy-related protein 8 (Atg8) family ${ }^{28}$ that displays a high degree of sequence homology with the autophagy marker light chain 3 (LC3). Kabeya et al. ${ }^{29}$ showed that GABARAP binds to autophagic vesicles in a similar manner to LC3 and may also be involved in autophagosome formation. By modulating autophagy, GABARAP has been reported play a key role in suppressing tumor growth ${ }^{30}$, inhibiting inflammation progression ${ }^{31}$, and regulating angiogenic activity ${ }^{32}$ via processes associated with vesicle transport, apoptotic cell death, and ROS generation. However, the role of GABARAP in regulating the differentiation fate of BMSCs via autophagy remains unclear.

Based on the studies described above, we hypothesized that the mediation of ROS generation and cellular autophagy by GABARAP may play pivotal roles in IL-1 $\beta$-induced BMSCs injury. This study is the first to demonstrate the regulatory effects of GABARAP on IL- $1 \beta$-induced cell apoptosis and BMSCs osteogenic differentiation in vitro, with a particular focus on the relationship of GABARAP with autophagy signaling pathways. Our longterm goal is to utilize GABARAP in bone regenerative therapy, such as improving the efficacy of BMSCs-based cellular regenerative therapies.

\section{Materials and methods}

BMSCs isolation and culture. Bone marrow was flushed from the femurs and tibias of 1-5-day-old New Zealand rabbits (Animal Resources Centre of Guangxi Medical University, Nanning, Guangxi, China) with a-modified Eagle's medium (a-MEM; Gibco, Thermo Fisher Scientific, Waltham, MA, USA) containing $10 \%$ (v/v) fetal bovine serum (FBS; Hyclone, Logan, UT, USA) and 1\% (v/v) penicillin/streptomycin (Solarbio, Beijing, China) using a 27 -gage syringe. The cell suspension was strained through a $70 \mu \mathrm{m}$ mesh filter and cultured in the same medium at $37^{\circ} \mathrm{C}$ under $5 \% \mathrm{CO}_{2}$. At $80-90 \%$ confluence, the cells were trypsinized, expanded, and used for different assays after their third passage. The animal protocol was approved by the Animal Ethical Committee of the Animal Resources Centre of Guangxi Medical University (ethic cord: 201902003). All animal experiments were performed in accordance with relevant guidelines and regulations. Meanwhile, animal studies were reported in compliance with the ARRIVE guidelines ${ }^{33,34}$ and complied with the principles of replacement, refinement and reduction (the $3 \mathrm{Rs}$ ).

BMSC multi-differentiation and cytological staining. Differentiated BMSCs were stimulated to undergo adipogenesis, osteogenesis, and chondrogenesis according to standard procedures ${ }^{35-37}$. Briefly, at $90 \%$ confluence, the BMSCs were incubated in a differentiation culture medium for a further 21 days. For adipocyte differentiation, the complete culture medium was supplemented with $1 \mathrm{mM}(10 \mathrm{nM})$ dexamethasone (Sigma, St Louis, MO, USA), $100 \mathrm{nM}(0.5 \mathrm{mM})$ indomethacin (Sigma), and $2 \mathrm{mM}$ insulin (Sigma). Adipogenesis was verified by Oil Red O staining (Beyotime, Beijing, China). To induce osteogenic and chondrogenic differentiation, the BMSCs were cultured with osteogenic medium $[10 \mathrm{mM} \beta$-glycerophosphate (Sigma), $50 \mathrm{mg} / \mathrm{ml}$ ascorbic acid 2-phosphate (Sigma), and $100 \mathrm{nM}$ dexamethasone] or chondrogenic medium $[50 \mu \mathrm{g} / \mathrm{mL}$ ascorbic acid 2-phosphate, $100 \mathrm{nM}$ dexamethasone, 1\% insulin-transferrin-selenium solution (Gibco), and $10 \mathrm{ng} / \mathrm{mL}$ TGF$\beta 1$ (PeproTech, Rocky Hill, PA, USA)], respectively. Alizarin Red (Sigma), ALP (Beyotime), and safranin O (Solarbio) staining were conducted to visualize calcium accumulation and evaluate chondrogenesis according to the manufacturer's suggested protocol. All staining results were viewed using an upright microscope (Olympus, Japan).

Inflammatory stimulation and the treatment of GABARAP and 3-MA in vitro. To determine the optimum His6-GABARAP (Boston Biochem, Cambridge, MA, USA) concentration for BMSCs proliferation, the cells were seeded in 96-well plates and treated with various concentrations of His6-GABARAP (0-200 nM) for 3 days. Viability was assessed using an MTT (3-(4,5-dimethylthiazol-2-yl)-2,5-diphenyltetrazolium bromide, Solarbio) assay, as described below. Based on the results, 30, 60, and $120 \mathrm{nM}$ His6-GABARAP were used to assess the effects of GABARAP on cell proliferation, viability, intracellular ROS, and multi-differentiation.

To induce osteogenesis, BMSCs were cultured in osteogenic medium in the presence of three doses of His6GABARAP $(30,60$, and $120 \mathrm{nM})$ for 21 days, after which osteogenic differentiation was determined by Alizarin Red and alkaline phosphatase (ALP) staining. Based on the levels of intracellular calcium accumulation, $60 \mathrm{nM}$ His6-GABARAP were selected as the most appropriate concentrations for observing the effect of His6-GABARAP on BMSCs osteogenic differentiation. Inflammation was stimulated by treating the cells with $10 \mathrm{ng} / \mathrm{mL}$ of IL- $1 \beta$, after which the cells were treated with His6-GABARAP with or without the autophagy inhibitor 3-MA (5 mM, Sigma) to clarify its relationship with the autophagy pathway in conventional culture or osteogenic differentiation.

Cell proliferation assay. BMSCs proliferation was assessed using MTT assays. One tenth of the volume of $5 \mathrm{mg} / \mathrm{mL}$ MTT solution was added to stimulated BMSCs per well and continuously incubated at $37^{\circ} \mathrm{C}$ for 
$4 \mathrm{~h}$ in the dark. The solution was then discarded, $150 \mu \mathrm{L}$ dimethyl sulfoxide (DMSO, Sigma) was added to each well, and the absorbance was measured at $490 \mathrm{~nm}$ using a Multiskan GO microplate reader (Thermo Fisher Scientific).

Hematoxylin and eosin (HE) staining: BMSCs cultured in 24-well plates were fixed for $30 \mathrm{~min}$ in $4 \%$ paraformaldehyde, stained with HE (Solarbio) according to the manufacturer's instructions, and dehydrated using an ethanol gradient. The slides were then sealed and examined using a microscope (Olympus).

Dual staining with fluorescein diacetate (FDA) and propidium iodide (PI). Harvested BMSCs were incubated with $2 \mu \mathrm{M}$ FDA (Invitrogen Life Technologies, CA, USA) and $2 \mu \mathrm{g} / \mathrm{L} \mathrm{PI} \mathrm{(Invitrogen)} \mathrm{for} 5$ min at $37^{\circ} \mathrm{C}$ in the dark. Live and dead cells were simultaneously evaluated via laser scanning confocal microscopy (Nikon A1, Japan).

Annexin V and PI staining. Differently treated BMSCs were harvested and re-suspended in Annexin V-FITC and PI working solutions (Thermo Fisher Scientific) and incubated at $4{ }^{\circ} \mathrm{C}$ in the dark. A total of $1 \times 10^{4}$ cells per sample were acquired by flow cytometry (BD Biosciences), and the percentage of apoptotic and live cells was assessed using FlowJo software (Tree Star Inc. Ashland, OR, USA).

Intracellular ROS measurement. Intracellular ROS were measured using a fluorescent 2,7-dichlorodihydrofluorescein diacetate (DCFH-DA) probe kit (Jiancheng) according to the manufacturer's protocol. Briefly, suitably treated BMSCs were incubated with $10 \mu \mathrm{M} \mathrm{DCFH-DA}$ at $37^{\circ} \mathrm{C}$ for $30 \mathrm{~min}$ and fluorescence was assessed by flow cytometry and FlowJo software analysis.

Monodansylcadaverine (MDC) staining. BMSCs were stained with $0.05 \mathrm{mM} \mathrm{MDC} \mathrm{(Sigma)} \mathrm{to} \mathrm{detect}$ autophagic vacuoles according to the manufacturer's instructions and assessed by laser scanning confocal microscopy, as described above.

Transmission electron microscopy (TEM). Harvested BMSCs were fixed for $24 \mathrm{~h}$ with $2.5 \%$ glutaraldehyde, incubated with $1 \%$ osmium tetroxide for $1 \mathrm{~h}$ in $4{ }^{\circ} \mathrm{C}$, and stained with $2 \%$ uranyl acetate. The BMSCs were then dehydrated using an acetone gradient and embedded in araldite. Sample sections were cut, stained with toluidine blue, and observed by TEM (Hitachi, Tokyo, Japan).

Autophagy flux measurement. StubRFP-sensGFP-LC3 lentiviruses were constructed by Genechem Co. (Shanghai, China). Primary first passage BMSCs were seeded in 6-well plates, cultured overnight, and transduced with the lentivirus in serum-free medium at a multiplicity of infection of 50 . After $12 \mathrm{~h}$, the media was replaced with complete $\alpha$-MEM and then the cells were treated with IL-1 $\beta$, His6-GABARAP, and 3-MA. Autophagic flux was observed using a laser scanning confocal microscope (Nikon America Inc., Melville, NY). stubRFP and sensGFP punctae were counted manually in at least 40 cells per sample.

Total RNA isolation and quantitative RT-PCR ( $q R T-P C R)$. Total RNA was isolated from BMSCs using Trizol reagent (Invitrogen), purified using a RNeasy mini kit (QIAGEN, Valencia, CA, USA), and reverse transcribed (1 mg total RNA per sample) using a Transcriptor First-strand cDNA synthesis kit (Roche, Basel, Switzerland). qRT-PCR was performed using SYBR green master mix on an Applied Biosystems 7500 Real Time Cycler (Applied Biosystems, CA, USA) under the following cycling conditions: $95^{\circ} \mathrm{C}$ for $10 \mathrm{~min}, 40$ cycles of $95^{\circ} \mathrm{C}$ for $15 \mathrm{~s}$ and $60^{\circ} \mathrm{C}$ for $1 \mathrm{~min}$, followed by a standard melting curve. Samples were assessed in triplicate, with gene expression assessed using the $2^{-\Delta \Delta \mathrm{CT}}$ method and normalized to GAPDH. The primer pairs for the target genes are listed in Table 1.

Western blotting. Protein was extracted from cell samples using RIPA Lysis Buffer (Beyotime) containing phenylmethanesulfonyl fluoride (PMSF) (Beyotime, China). Equal protein quantities (60 $\mu \mathrm{g}$ ) were denatured using SDS-PAGE loading buffer, separated by 6-15\% polyacrylamide gel electrophoresis, and transferred to PVDF membranes (Millipore, Billerica, MA, USA). Prior to hybridization with antibodies, the blots were cut according to the weight of target proteins. Blots were probed at $4{ }^{\circ} \mathrm{C}$ overnight using primary antibodies against mTOR, p-mTOR, LC3, (1:1000, Cell Signaling Technology, Beverly, MA, USA), and GAPDH (1:10,000, Cell Signaling Technology). The blots were washed three times with PBST and then probed with an appropriate DyLight ${ }^{\mathrm{sm}}$ 800 4X PEG conjugated secondary antibody (1:10,000, Cell Signaling Technology) for $1 \mathrm{~h}$. Protein bands were identified using an Odyssey Infrared Imaging System and protein levels were quantified relative to the GAPDH loading control using ImageJ software (NIH, Bethesda, Maryland, USA).

Statistical analysis. Data were presented as the mean \pm SD and assessed using SPSS version 22.0. Parametric data were compared by one-way analysis of variance (ANOVA) with least significant difference (LSD) post-hoc tests, while non-parametric data were assessed by Mann-Whitney $U$ tests. $p$ values of $<0.05$ were considered statistically significant.

\section{Results}

GABARAP increases BMSCs viability and proliferation by inhibiting intracellular ROS generation. BMSCs were treated with varying concentrations of His6-GABARAP for 3 days, with no apparent toxicity observed for doses of 0-50 nM (Fig. 1). BMSCs exposed to 40, 60, 70, and $80 \mathrm{nM}$ of His6-GABARAP 


\begin{tabular}{|c|c|c|}
\hline Gene & Primer & Primer sequence $\left(5^{\prime}-3^{\prime}\right)$ \\
\hline \multirow{2}{*}{ Glyceraldehyde-3-phosphate dehydrogenase (GAPDH) } & Forward & AGACACGATGGTGAAGGTCG \\
\hline & Reverse & TGCCGTGGGTGGAATCATAC \\
\hline \multirow{2}{*}{ BCL2 associated X (BAX) } & Forward & CGTGCGATCTCCAAGCACTC \\
\hline & Reverse & CCAAGTTATCAGGGGTCCGA \\
\hline \multirow{2}{*}{ B cell leukemia-2 (Bcl-2) } & Forward & TTTGAGTTCGGTGGGGTCAT \\
\hline & Reverse & GGCCGTACAGTTCCACGAAG \\
\hline \multirow{2}{*}{ caspase-3 } & Forward & GAACAACGAAACCTCCGTGG \\
\hline & Reverse & TCCCAGAGTCCATTGATTTGC \\
\hline \multirow{2}{*}{ Interleukin-6 (IL-6) } & Forward & AAGAAAACACCAGGGTCAGC \\
\hline & Reverse & TGGTTTTTCTGCTGCAGGTTC \\
\hline \multirow{2}{*}{ Tumor necrosis factor- $\alpha$ (TNF- $\alpha$ ) } & Forward & AGCCCACGTAGTAGCAAACC \\
\hline & Reverse & TGAGTGAGGAGCACGTAGGA \\
\hline \multirow{2}{*}{ Matrix metallopeptidase-13 (MMP-13) } & Forward & TTTCTCGTTGCTGCCCATGA \\
\hline & Reverse & GGGTGTTTAGGGTTGGGGTC \\
\hline \multirow{2}{*}{ Manganese superoxide dismutase (MnSOD) } & Forward & ACAAACCTGAGCCCTAACGG \\
\hline & Reverse & AAGCGTGTTCCCACACATCA \\
\hline \multirow{2}{*}{ Superoxide dismutase [Cu-Zn]-like (CuZnSOD) } & Forward & GCCGCTGCGGAGTCAT \\
\hline & Reverse & TCGGTCAGTCCTGTTATGCG \\
\hline \multirow{2}{*}{ Autophagy related 5 (Atg5) } & Forward & GACGACGACTGAACGACCTT \\
\hline & Reverse & GAGCAATTGCGGAAGGACAC \\
\hline \multirow{2}{*}{ Autophagy related 7 (Atg7) } & Forward & TTCGACAAATGCACCGCTTG \\
\hline & Reverse & ACTCAGACGGTCTCCTCGTC \\
\hline \multirow{2}{*}{ Beclin-1 } & Forward & CCAGGTCAAACCAGGAGACC \\
\hline & Reverse & CCCCGATCAGAGTGAAGCTG \\
\hline \multirow{2}{*}{ Mechanistic target of rapamycin kinase (mTOR) } & Forward & GGCAAGATGCTGGGGACC \\
\hline & Reverse & CGCGTTGACTCTTCCTGACT \\
\hline \multirow{2}{*}{ Alkaline phosphatase (ALP) } & Forward & TCTCTGAGCCTCGTGAAACG \\
\hline & Reverse & GCTCACСТCAGACACACCTC \\
\hline \multirow{2}{*}{ Collagen type I (Col I) } & Forward & CAGCGGCTCCCCATTTTCTA \\
\hline & Reverse & ATCTCAGCTCGCATAGCACC \\
\hline \multirow{2}{*}{ Osteocalcin (OCN) } & Forward & AGAGTCTGGCAGAGGCTCA \\
\hline & Reverse & CAGGGGATCCGGGTAAGGA \\
\hline \multirow{2}{*}{ Osteopontin (OPN) } & Forward & CACAGCGTGGAAACCCAAAG \\
\hline & Reverse & TGGCCTCGCGCTTATATTGT \\
\hline \multirow{2}{*}{ Peroxisome proliferator-activated receptor gamma $(\operatorname{PPAR} \gamma)$} & Forward & TCACAAGAGGTGACCCAATG \\
\hline & Reverse & CCATCCTTCACAAGCATGAA \\
\hline \multirow{2}{*}{ Fatty acid binding protein 4 (FABP4) } & Forward & TTCCTGTCGTCTGCGGTGATT \\
\hline & Reverse & GATGCCTTTGTGGGAACCTGG \\
\hline \multirow{2}{*}{ Collagen type II (Col II) } & Forward & ACGCTCAAGTCGCTGAACAACC \\
\hline & Reverse & ATCCAGTAGTCTCCGCTCTTCCAC \\
\hline \multirow{2}{*}{ ACAN } & Forward & CTGATCCACTGTCCAAGCACCATG \\
\hline & Reverse & ATCCACGCCAGGCTCCACTC \\
\hline
\end{tabular}

Table 1. Primer sequences used in qRT-PCR experiments.

displayed significantly higher proliferation, with the most dramatic increase seen for $60 \mathrm{nM}$; therefore, 30, 60, and $120 \mathrm{nM}$ of His6-GABARAP were selected for the subsequent experiments.

First, we carried out HE staining to evaluate changes in cell morphology after His6-GABARAP treatment for 7 days. His6-GABARAP did not affect the typical fibroblastic cell morphology of cultured BMSCs but did increase the cell density compared to the normal group (Fig. 2B), as confirmed by MTT assays. As shown in Fig. 2C, His6-GABARAP concentrations of 30, 60, and $120 \mathrm{nM}$ increased cell proliferation at days 1, with $60 \mathrm{nM}$ of His6-GABARAP particularly increasing proliferation on days 3 (21.8 $\pm 5.2 \%$ higher than the control group), $5(8.9 \pm 1.4 \%$ higher than the control group), and 7 ( $12.4 \pm 1.6 \%$ higher than the control group).

Next, we examined the effects of His6-GABARAP on BMSCs viability by Annexin V-FITC, PI, and FDA/ PI staining. BMSCs treated with 30 and $60 \mathrm{nM}$ of His6-GABARAP showed a significantly higher proportion of viable cells after 7 days of treatment (Fig. 2E), with the highest percentage of live cells observed in the $60 \mathrm{nM}$ His6-GABARAP-treated group (94.4 $\pm 0.7 \%)$. Staining with FDA/PI dyes, which differentiate between dead and live cells, revealed that BMSCs exposed to 30 and $60 \mathrm{nM}$ of His6-GABARAP showed significantly higher viability after 1, 3, 5, and 7 days of treatment (Fig. 2A), with the optimal effects observed at $60 \mathrm{nM}$. After 7 days, 30 


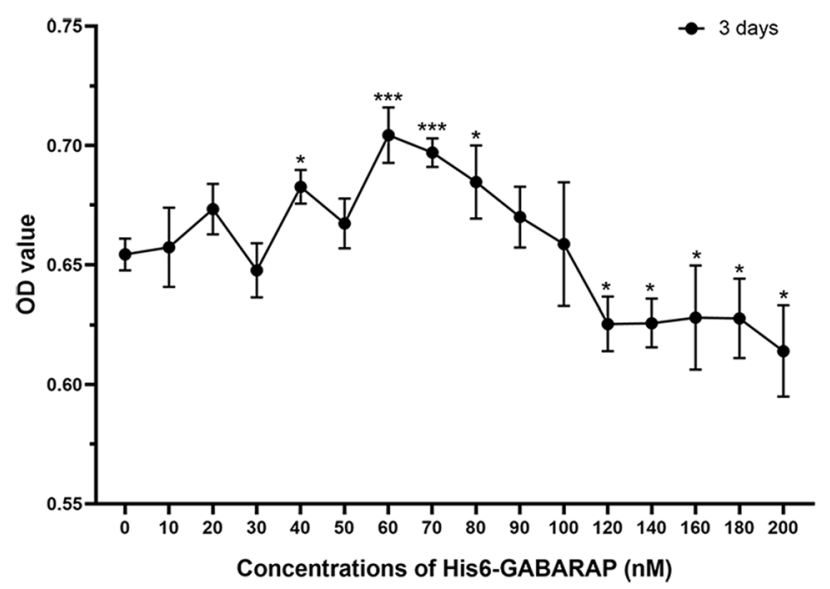

Figure 1. Edification the cytotoxicity of His6-GABARAP on BMSCs. MTT assay was applied after co-cultured with varying concentrations of His6-GABARAP for 3 days. Values are the means $\pm \mathrm{SD}\left({ }^{\star} p<0.05,{ }^{* \star *} p<0.001\right.$ indicate the significant difference relative to the normal group, $n=3$ ).

and $60 \mathrm{nM}$ of His6-GABARAP also decreased intracellular ROS levels to $21.8 \pm 1.9$ and $9.4 \pm 1.5 \%$, respectively (Fig. 2D). Taken together, these results demonstrate that GABARAP increases BMSCs viability by blocking intracellular ROS generation.

GABARAP protects BMSCs against IL-1 $\beta$-induced apoptosis, inflammation, and autophagy inhibition. Next, we investigated the protective effects of His6-GABARAP in BMSCs treated with $10 \mathrm{ng} /$ $\mathrm{mL}$ of IL-1 $\beta$. When exposed to IL-1 $\beta$ alone, BMSCs displayed a flattened, rounded shape; however, the cells maintained a fibroblastic morphology when co-treated with His6-GABARAP (Fig. 3A). At a concentration of $60 \mathrm{nM}$, His6-GABARAP significantly increased the rate of cell proliferation to $12.6 \pm 5.9,29.5 \pm 5.8,24.7 \pm 8.5$, and $33.8 \pm 10.9 \%$ higher than the IL- $1 \beta$-treated group on days $1,3,5$, and 7 , respectively (Fig. 3C). Flow cytometry (Fig. 3E) and FDA/PI (Fig. 3B) staining also showed that His6-GABARAP treatment could increase the percentage of live cells and decrease the level of apoptosis induced by IL- $1 \beta$. Similarly, His6-GABARAP was able to reverse the up-regulation of the pro-apoptotic gene caspase- 3 in IL- $1 \beta$-treated cells (Fig. 5A) and increased the gene expression ratio of $\mathrm{Bcl}-2 / \mathrm{BAX}$ (Fig. 5B-D). Moreover, His6-GABARAP-treated cells displayed dramatically lower ROS levels than the IL-1 $\beta$-treated group (Fig. 3D).

To determine whether His6-GABARAP functioned by inhibiting inflammation, we performed qRT-PCR to detect the expression levels of inflammatory factors such as IL-6 (Fig. 5E), TNF- $\alpha$ (Fig. 5F), and MMP-13 (Fig. 5G). Compared to the vehicle-treated cells, His6-GABARAP treatment effectively suppressed the IL-1 $\beta$ induced increase in inflammatory cytokine levels and restored the expression of antioxidant-specific markers including MnSOD (Fig. 5H) and CuZnSOD (Fig. 5I) in BMSCs. To investigate whether His6-GABARAP triggered autophagy in BMSCs under inflammatory conditions, we measured Atg5, Atg7, LC3, Beclin-1, and mTOR expression levels by qRT-PCR and western blotting, finding that LC3-II/LC3-I, Atg5, Atg7, and Beclin-1 expression levels were dramatically lowered by IL- $1 \beta$ treatment and reversed when co-cultured with His6-GABARAP (Figs. 4A and 5K). Meanwhile, the expression of mTOR was suppressed by His6-GABARAP as compared with IL-1 $\beta$-treated group (Figs. $4 \mathrm{~A}$ and $5 \mathrm{~K}$ ). Taken together, these results demonstrate that GABARAP alleviates pathological changes in BMSCs by blocking intracellular ROS generation, inflammation, and apoptosis, and that these effects are likely mediated via autophagy activation.

GABARAP up-regulates cell survival by targeting autophagy mechanisms. To clarify the role of autophagy in the protective effects of His6-GABARAP in BMSCs viability and inflammation, we treated IL-1 $\beta$ induced BMSCs with His6-GABARAP with or without 3-MA. MTT assays confirmed that 3-MA negatively affected the proliferation of IL-1 $\beta$-treated BMSCs and effectively blocked the promotion of cell proliferation by His6-GABARAP (Fig. 4B). By detecting the expression of apoptosis-, antioxidant-, and inflammation-related cytokines in BMSCs, we found that 3-MA prevented the His6-GABARAP-mediated inhibition of cell apoptosis, oxidative stress, and inflammation (Fig. 5A-I), indicating that 3-MA treatment has an inhibitory effect on BMSCs viability that is promoted by His6-GABARAP in an inflammatory microenvironment.

To explore the role of autophagy in the interaction between 3-MA and His6-GABARAP, we detected the autophagy-related cytokines Atg5, Atg7, LC3, Beclin-1, and mTOR. As shown in Fig. 5J and 5K, 3-MA decreased Atg5, Atg7, and Beclin-1 expression levels and the LC3-II/LC3-I ratio which were increased by His6-GABARAP, along with the number of MDC-labeled autophagic vacuoles in IL- $1 \beta$-stimulated BMSCs (Fig. 4D). mTOR expression levels were again elevated by 3-MA as compared with His6-GABARAP-treated group (Fig. 5J and $5 \mathrm{~K})$. However, the downregulation of autophagy markers and the presence of numerous autophagic vacuoles are static inhibitors of autophagy. Thus, to detect autophagic flux we transduced BMSCs with RFP-GFP-LC3 to simultaneously track autophagosomes (yellow punctae that are a combination of red and green fluorescence) 
A<smiles>[Mg][Mg][AlH2]</smiles>

1 days
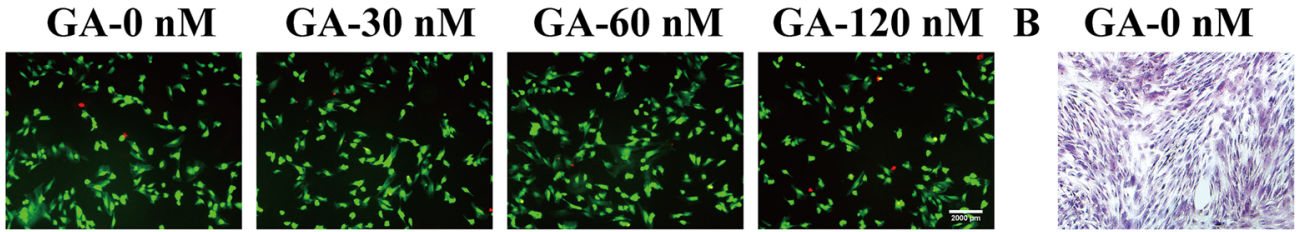

GA-30 nM
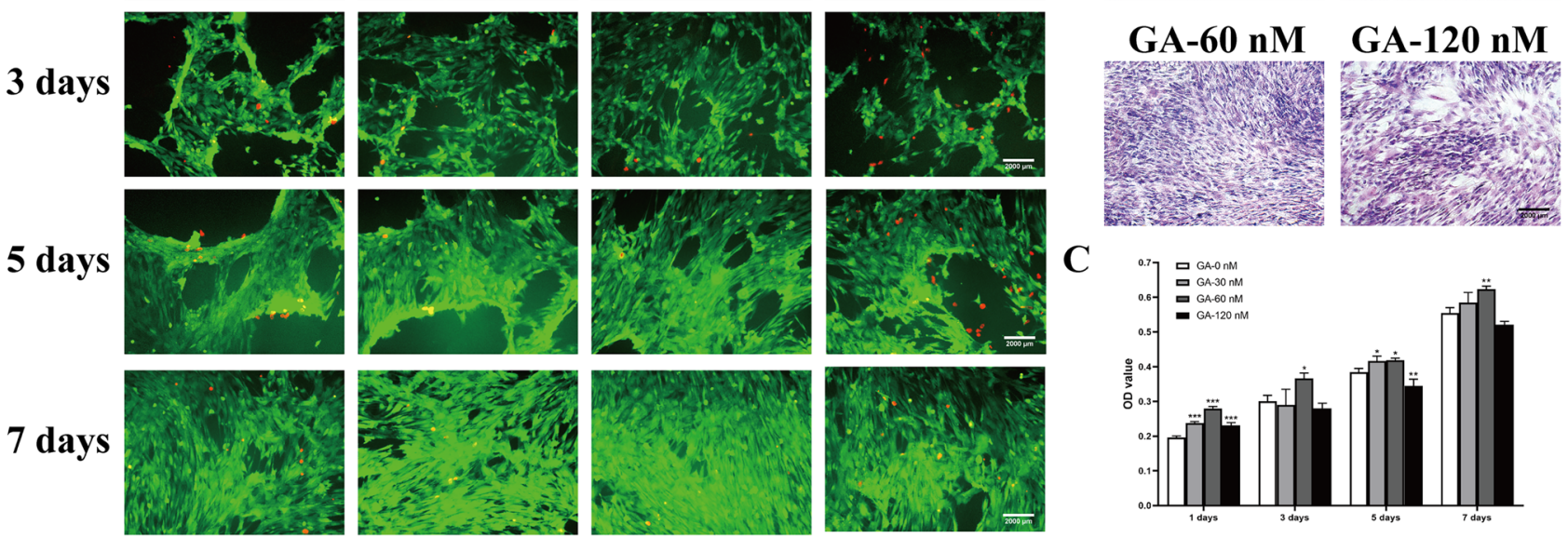

C

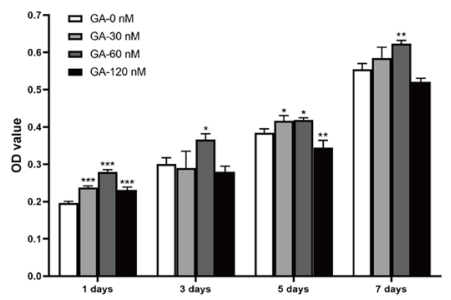

D

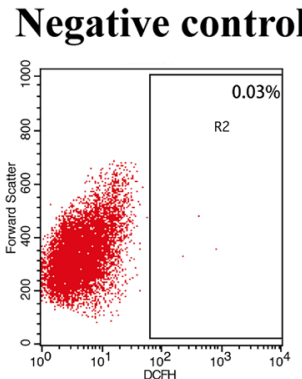

GA-0 nM
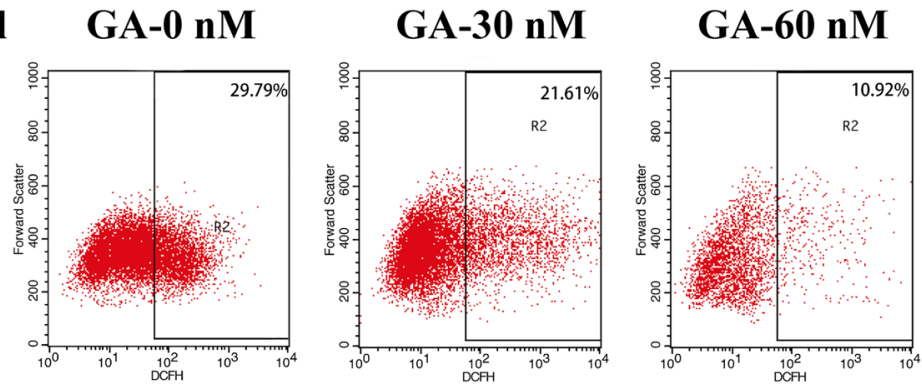

GA-120 nM

$\mathbf{E}$
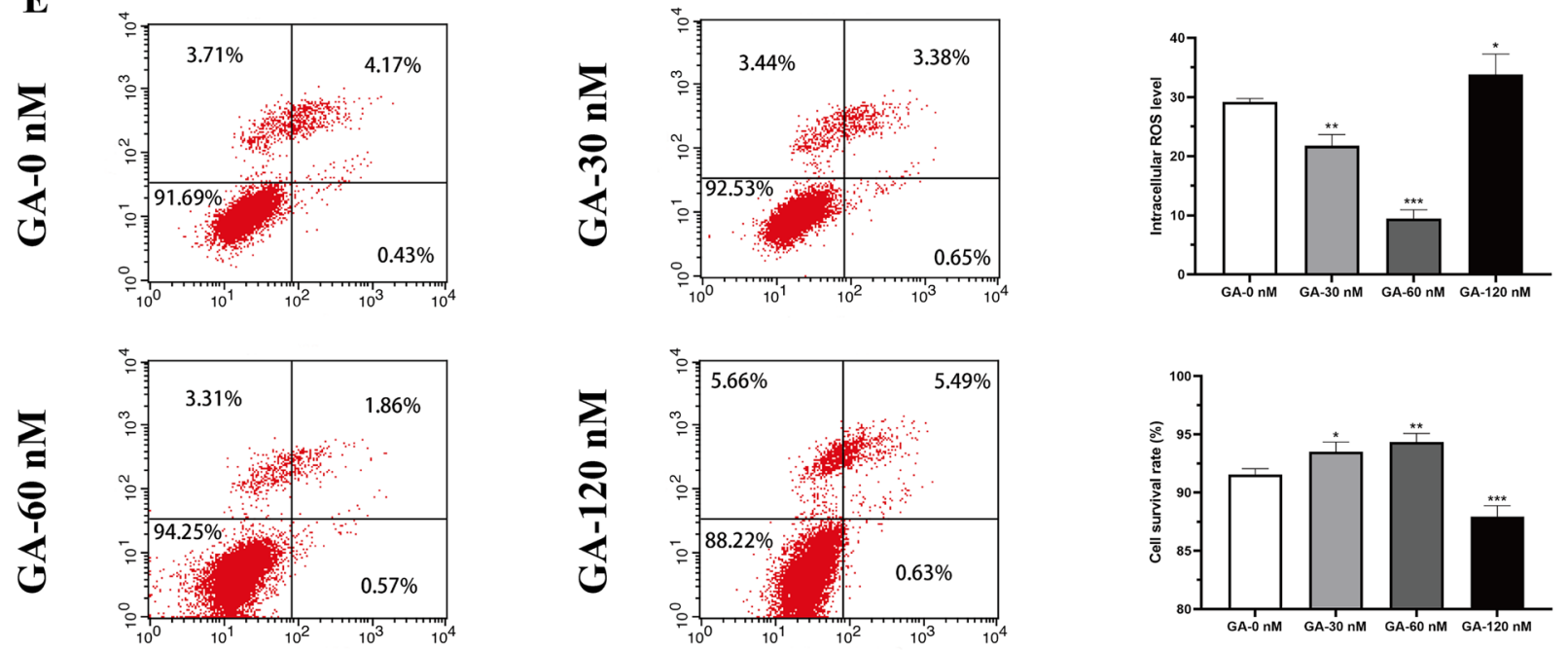

Figure 2. Promotion effects of His6-GABARAP on normal BMSCs. MTT assay (C) and FDA/PI staining (A) were applied to detect the cytotoxicity and viability of His6-GABARAP at days 1,3,5, and 7. Cells were treated with various concentrations of His6-GABARAP for 7 days. (B) H\&E stained for cell morphology. (D) Flow cytometry for intracellular ROS. (E) Quantitative flow cytometry for viability. GA-0 nM (without His6-GABARAP); GA-30 nM (with $30 \mathrm{nM}$ His6-GABARAP); GA-60 nM (with $60 \mathrm{nM}$ His6-GABARAP); GA-120 nM (with $120 \mathrm{nM}$ His6-GABARAP). Values are the means $\pm \mathrm{SD}, \mathrm{n}=3 .{ }^{*} \mathrm{p}<0.05,{ }^{* *} \mathrm{p}<0.01,{ }^{* * *} \mathrm{p}<0.001$ relative to the normal group. Scale bar, $2000 \mu \mathrm{m}$.

and autophagolysosomes (red punctae). His6-GABARAP treatment significantly increased the proportion of autophagolysosomes in the cytoplasm of IL- $1 \beta$-treated BMSCs (Fig. $4 \mathrm{E}$ ), indicating that autophagy was induced. In addition, TEM confirmed the increased number of autophagic vesicles and autolysosomes 


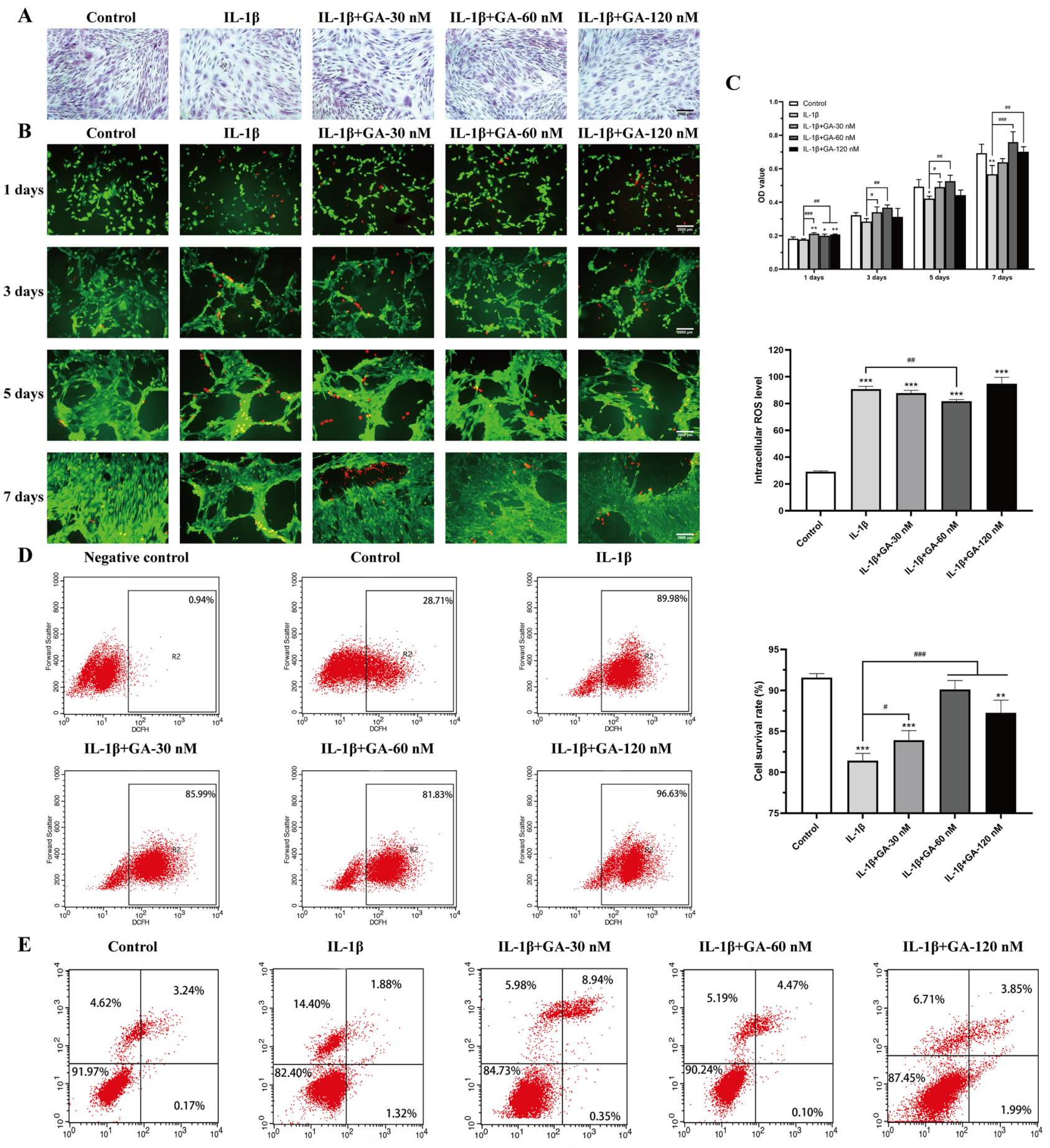

Figure 3. Protective effects of His6-GABARAP on IL-1 $\beta$-induced BMSCs. H\&E (A), FDA/PI staining (B), MTT assay $(\mathbf{C})$, and Flow cytometry $(\mathbf{D}, \mathbf{E})$ were applied for cell morphology, viability, proliferation, and intracellular ROS. Control (without IL-1 $\beta$ ); IL-1 $\beta$ (with $10 \mathrm{ng} / \mathrm{mL}$ IL-1 $\beta$ ); IL-1 $\beta+$ GA-30 nM (with $10 \mathrm{ng} / \mathrm{mL}$ IL-1 $\beta$ and $30 \mathrm{nM}$ His6-GABARAP); IL-1 $\beta+$ GA-60 nM (with $10 \mathrm{ng} / \mathrm{mL}$ IL-1 $\beta$ and $60 \mathrm{nM}$ His6-GABARAP); IL-1 $\beta+$ GA-120 nM (with $10 \mathrm{ng} / \mathrm{mL}$ IL- $1 \beta$ and $120 \mathrm{nM}$ His6-GABARAP). Values are presented as means \pm SD, $\mathrm{n}=3 .{ }^{*} p<0.05,{ }^{* *} p<0.01,{ }^{* * *} p<0.001$ relative to the control group; $\# p<0.05, \# \# p<0.01, \# \# p<<0.001$ relative to the IL-1 $\beta$ group. Scale bar, $2000 \mu \mathrm{m}$.

in the His6-GABARAP-treated BMSCs (Fig. 4C). As expected, 3-MA partially inhibited the autophagic flux and autophagolysosome-inducing effects of His6-GABARAP (Fig. 4C and E). Taken together, these findings suggest that His6-GABARAP activates autophagic flux in IL-1 $\beta$-treated BMSCs as the underlying basis of its regenerative effects. 


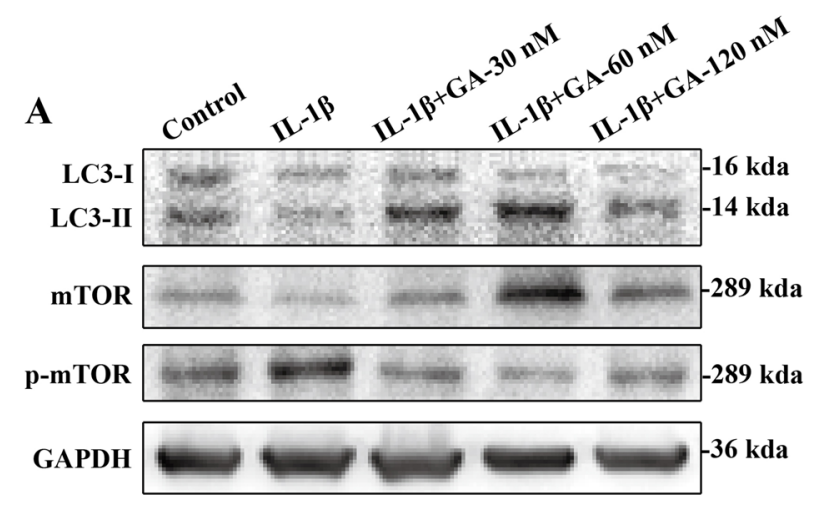

B

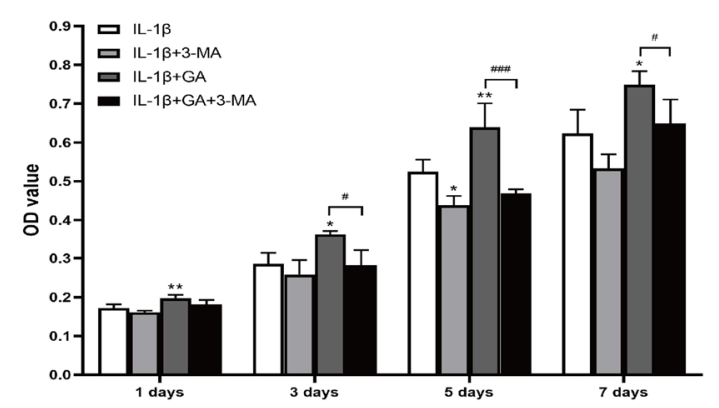

C

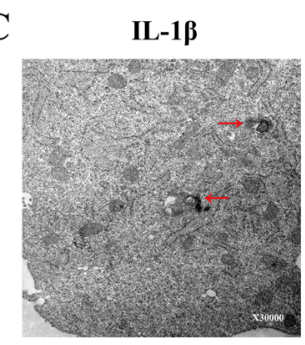

D
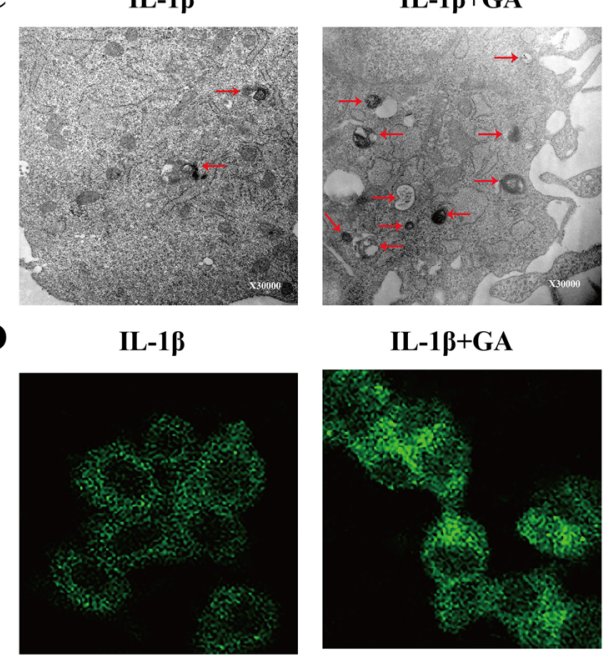
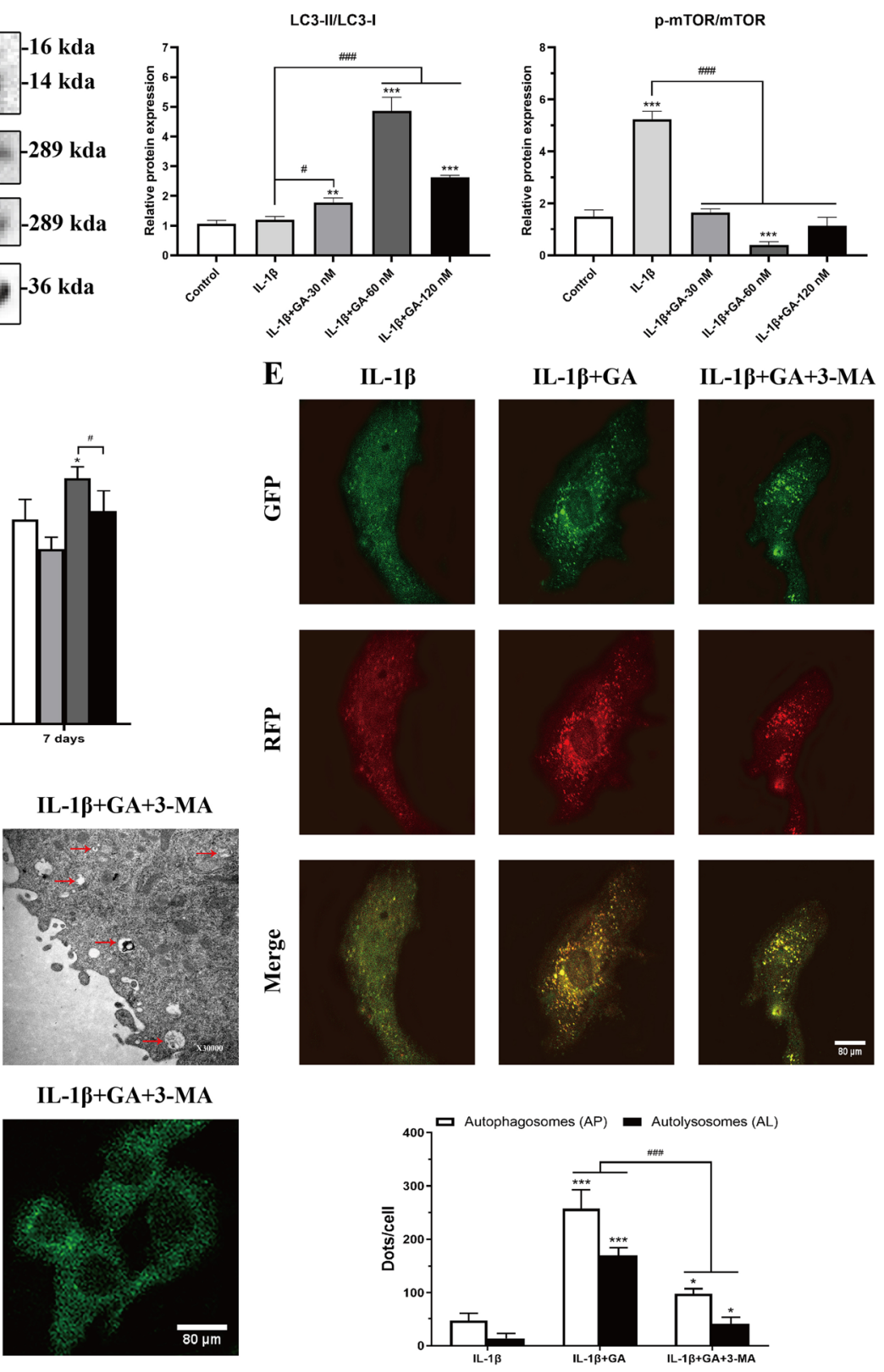

Figure 4. Effects of His6-GABARAP on autophagic flux in BMSCs in vitro. (A) Western blot of LC3, mTOR, and p-mTOR in different groups were detected respectively and the semi-quantitative analysis of LC3 II/I ratio and mTOR/p-mTOR are shown. (B) MTT assay were applied to detect the cell proliferation at days 1, 3, 5, and 7. (C) TEM images of the autophaghic change in BMSCs. Single arrow: autophagolysosome and autophagosome with double membrane structure. Original magnification $\times 30,000$. (D) Representative pictures of MDC staining. Scale bar, $80 \mu \mathrm{m}$. (E) Representative pictures of immunofluorescent BMSCs expressing mRFP-GFP-LC3. GFP dots are green, and mRFP dots are red. Scale bar, $80 \mu \mathrm{m}$. And Semi-quantitative analysis of autophagosomes (AP; yellow dots in merged images) and autolysosomes (AL; red only dots in merged images). Control (without IL-1 $\beta$ ); IL-1 $\beta$ (with $10 \mathrm{ng} / \mathrm{mL}$ IL-1 $\beta$ ); IL-1 $\beta$ +3-MA (with $10 \mathrm{ng} / \mathrm{mL}$ IL-1 $\beta$ and 3-MA); IL-1 $\beta$ + GA (with $10 \mathrm{ng} /$ mL IL-1 $\beta$ and $60 \mathrm{nM}$ His6-GABARAP); IL-1 $\beta+\mathrm{GA}+3-\mathrm{MA}$ (with $10 \mathrm{ng} / \mathrm{mL}$ IL-1 $\beta, 60 \mathrm{nM}$ His6-GABARAP, and 3-MA). Values are presented as means $\pm \mathrm{SD}, \mathrm{n}=3 .{ }^{\star} p<0.05,{ }^{* *} p<0.01,{ }^{\star * *} p<0.001$ relative to the control group; $\# p<0.05$, \#\# $p<0.01$, \#\#\# $p<0.001$ relative to the IL- $1 \beta$ group.

GABARAP activation modulates chondrogenic differentiation and adipogenesis in vitro. BMSCs can differentiate into multiple cell types, including chondrocytes and adipocytes; therefore, to determine the effect of His6-GABARAP on BMSCs differentiation, we cultured MSCs in chondrogenesis or adipogenesis medium. As verified by very low levels of regulation in the Oil Red O stain, His6-GABARAP 


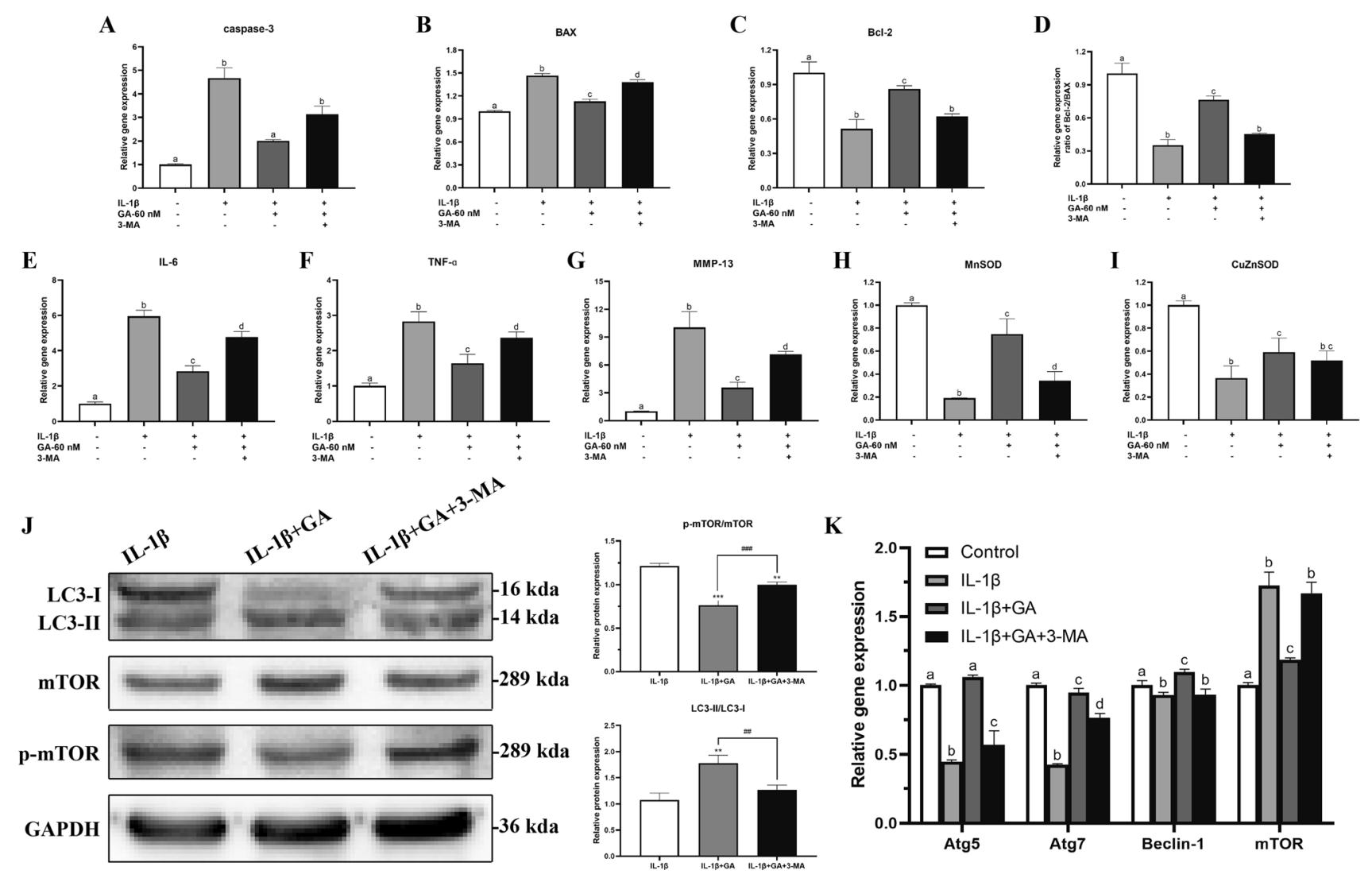

Figure 5. Effect of His6-GABARAP on the treatment of IL- $1 \beta$-induced BMSCs. (A-I, and K) qRT-PCR was used to analyze the gene expression levels of caspase-3, BAX, Bcl-2, Bcl-2/BAX ratio, TNF- $\alpha$, IL-6, MMP-13, MnSOD, CuZnSOD, Atg5, Atg7, Beclin-1, and mTOR in vitro. (J) Western blot was used to analyze the protein expression of LC3, mTOR, and p-mTOR. Values are presented as means $\pm \mathrm{SD}, \mathrm{n}=3$. Bars with different letters are significantly different from each other at $p<0.05$ and those with the same letter exhibit no significant difference. ${ }^{* *} p<0.01,{ }^{* *} p<0.001$ relative to the IL- $1 \beta$ group; $\# p<0.01$, \#\#\# $p<0.001$ relative to the IL- $1 \beta+$ GA group.

did not affect BMSCs adipogenesis (Fig. 6A). Meanwhile, the expression level of adipogenic differentiationconnected genes, including FABP4 and PPAR $\gamma$, also showed that His6-GABARAP had little effect on BMSCs adipogenesis (Fig. 6I and J). However, the results of Safranin O staining demonstrated that His6-GABARAP inhibited chondrocyte formation (Fig. 6B). At the genetic level, genes expression level of chondrogenesis markers Col II and ACAN were significantly decreased by His6-GABARAP (Fig. 6K and L), which demonstrated that chondrogenesis differentiation was markedly reduced in His6-GABARAP-treated BMSCs compared to WT differentiated BMSCs. These results may provide evidence for the additional role of His6-GABARAP in BMSCs chondrogenesis and adipogenesis.

GABARAP maintains BMSC osteogenic differentiation in normal and inflammatory microenvironments. Osteogenic differentiation was induced by culturing BMSCs in osteogenic media with or without 30, 60, and $120 \mathrm{nM}$ of His6-GABARAP. As shown in Fig. 6C and D, ALP and Alizarin Red staining after 21 days indicated that His6-GABARAP stimulated BMSCs osteogenesis, with $60 \mathrm{nM}$ of His6-GABARAP causing the most significant change in calcium deposition. We then quantified the mRNA levels of the osteoblast marker genes Col I, ALP, OCN, and OPN after 7, 14, and 21 days (Fig. 6E-H). His6-GABARAP increased Col I, OPN, ALP, and OCN transcription, particularly at a treatment concentration of $60 \mathrm{nM}$ His6-GABARAP. Therefore, we used $60 \mathrm{nM}$ of His6-GABARAP for subsequent experiments.

In the presence of IL-1 $\beta, 60 \mathrm{nM}$ of His6-GABARAP stimulated denser deposition of Alizarin Red and ALP staining after 7, 14, and 21 days of osteogenic differentiation culture (Fig. 7A and B). Moreover, IL-1 $\beta$-treated BMSCs had dramatically lower mRNA expression of the osteoblastic differentiation factors Col I, ALP, OCN, and OPN (Fig. 7C-F); however, treatment with $60 \mathrm{nM}$ of His6-GABARAP yielded higher COL I, ALP, OCN, and OPN mRNA expression levels than in IL-1 $\beta$ treated BMSCs. Taken together, these results suggest that His6GABARAP can maintain their BMSCs osteogenic differentiation under inflammatory conditions.

Autophagy plays a role in GABARAP-promoted BMSCs osteogenic differentiation. To determine the mechanisms responsible for the increased osteoblastogenesis observed in His6-GABARAP-treated BMSCs under inflammatory conditions, we used 3-MA to suppress autophagy. Interestingly, inhibiting 

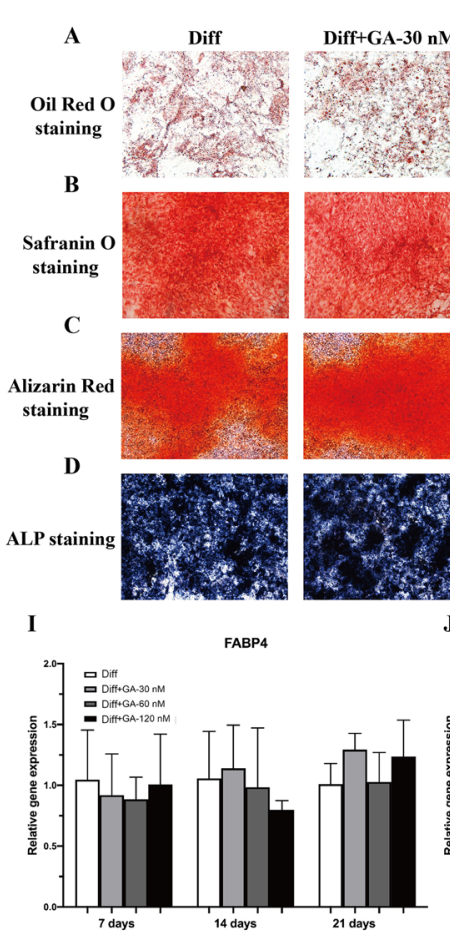
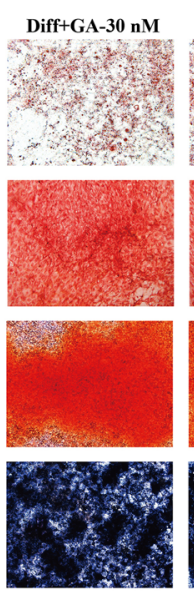

$\mathbf{J}$
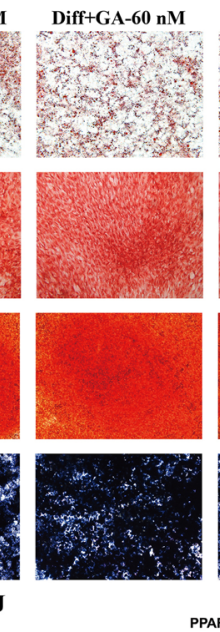

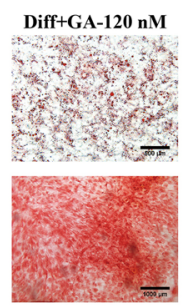

E
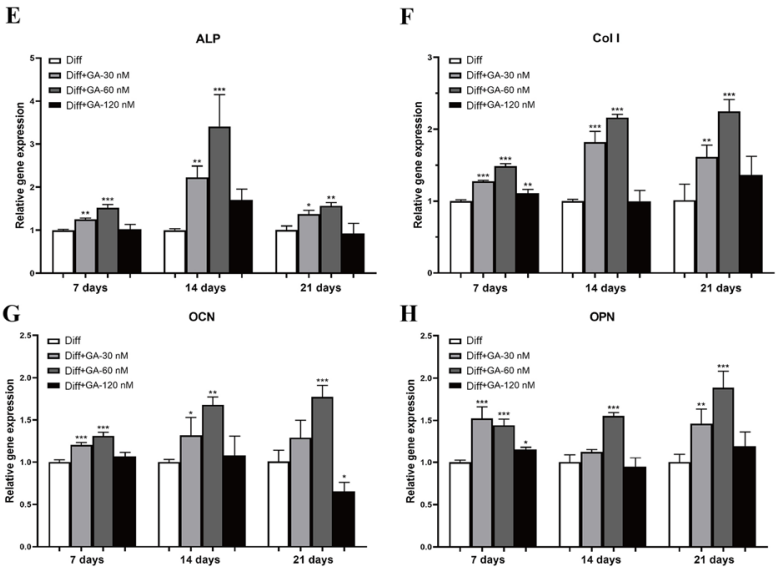

K

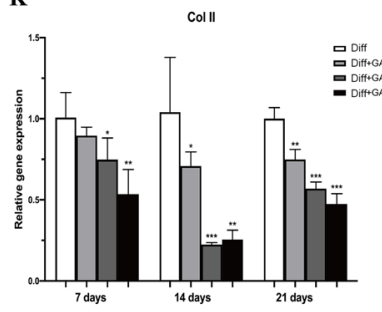

Figure 6. The effect of His6-GABARAP on BMSCs differentiation. Adipogenesis and chondrogenesis differentiation of BMSCs were verified by Oil Red O (A) and safranin O (B) staining, respectively, in 21 days. Qualitative assessment of calcium deposition in BMSCs was stained by Alizarin Red (C) and ALP (D) staining in 21 days. qRT-PCR was used to analyze the gene expression levels of ALP (E), Col I (F), OCN (G), OPN (H), FABP4 (I), PPAR $\gamma(\mathbf{J})$, Col II (K), and ACAN (L) during differentiation. Diff (with corresponding differentiation); Diff + GA-30 nM (with corresponding differentiation and $30 \mathrm{nM}$ His6-GABARAP); Diff + GA-60 nM (with corresponding differentiation and $60 \mathrm{nM}$ His6-GABARAP); Diff + GA-120 nM (with corresponding differentiation and $120 \mathrm{nM}$ His6-GABARAP). Values are the means $\pm \mathrm{SD}, \mathrm{n}=3 .{ }^{*} p<0.05$, ${ }^{* *} p<0.01,{ }^{* *} p<0.001$ relative to the Diff group. Scale bar, 500\&1000 $\mu \mathrm{m}$.

autophagy with 3-MA enhanced the inhibitory effects of IL-1 $\beta$ in BMSCs osteogenic differentiation and abrogated the restorative effects of His6-GABARAP in vitro (Fig. 7A-F). Similarly, culturing BMSCs with His6GABARAP in presence of IL-1 $\beta$ under osteogenic differentiation significantly increased the gene and protein expression of Atg5, Atg7, Beclin-1, and the ratio of LC3-II/LC3-I (Fig. 7G-J). Moreover, BMSCs co-cultured with 3-MA and His6-GABARAP displayed significantly lower levels of autophagy activation, as evidenced by downregulated Atg5, Atg7, Beclin-1, and LC3-II/LC3-I expression levels and upregulated expression level of mTOR (Fig. 7G-K). Thus, these results suggest that autophagy activation plays a critical role in His6-GABARAP promoting BMSCs osteogenic differentiation.

\section{Discussion}

Previously, study reported that weekly intra-articular His6-GABARAP administration effectively promotes the therapeutic effect of BMSC-derived chondrocytes in OA lesions ${ }^{38}$; however, the initiation and progression of OA involves a variety of pathological mechanisms, including the loss of bone mass in subchondral bone ${ }^{39,40}$ and synovium lesions ${ }^{41}$. Meanwhile, its specific therapeutic mechanisms, particularly the associations between GABARAP and BMSCs or chondrocytes under inflammatory conditions, remain unknown. In present study, it had been demonstrated that autophagy plays an important role in promoting BMSCs osteogenic differentiation and restricts intracellular ROS generation ${ }^{27}$. Furthermore, it was also revealed that autophagy activation could enhance BMSCs proliferation ${ }^{42}$. Consistently, our pilot study also found that GABARAP could mediate BMSCs cell viability and osteogenic differentiation, which are inhibited by the pro-inflammatory factor IL- $1 \beta$; thus, it may provide a potential application of GABARAP in OA repairment by promoting subchondral bone remodeling.

The induction of cellular ROS generation and activation of apoptosis signaling by IL-1 $\beta$ has been increasingly recognized to play a vital role in cell stress ${ }^{43,44}$, and followed to induce senescence and apoptosis in various cell types, including $\mathrm{BMSCs}^{6}$, chondrocytes ${ }^{45}$, and osteocytes ${ }^{46}$, thus, IL-1 $\beta$ with significant pathological implications in the progression of many inflammatory diseases. Consistently, we found that IL-1 $\beta$ in the microenvironment may serve as an important modulator of BMSCs viability and proliferation and increase the expression of the inflammatory markers IL-6, TNF-a, and MMP-13. However, His6-GABARAP treatment effectively maintained cell survival, restored the fibroblastic-like morphology of BMSCs, and downregulated pro-inflammatory cytokines and apoptosis-related markers. Furthermore, GABARAP not only suppressed inflammatory damage in BMSCs, but also enhanced BMSC proliferation and viability without IL-1 $\beta$. 

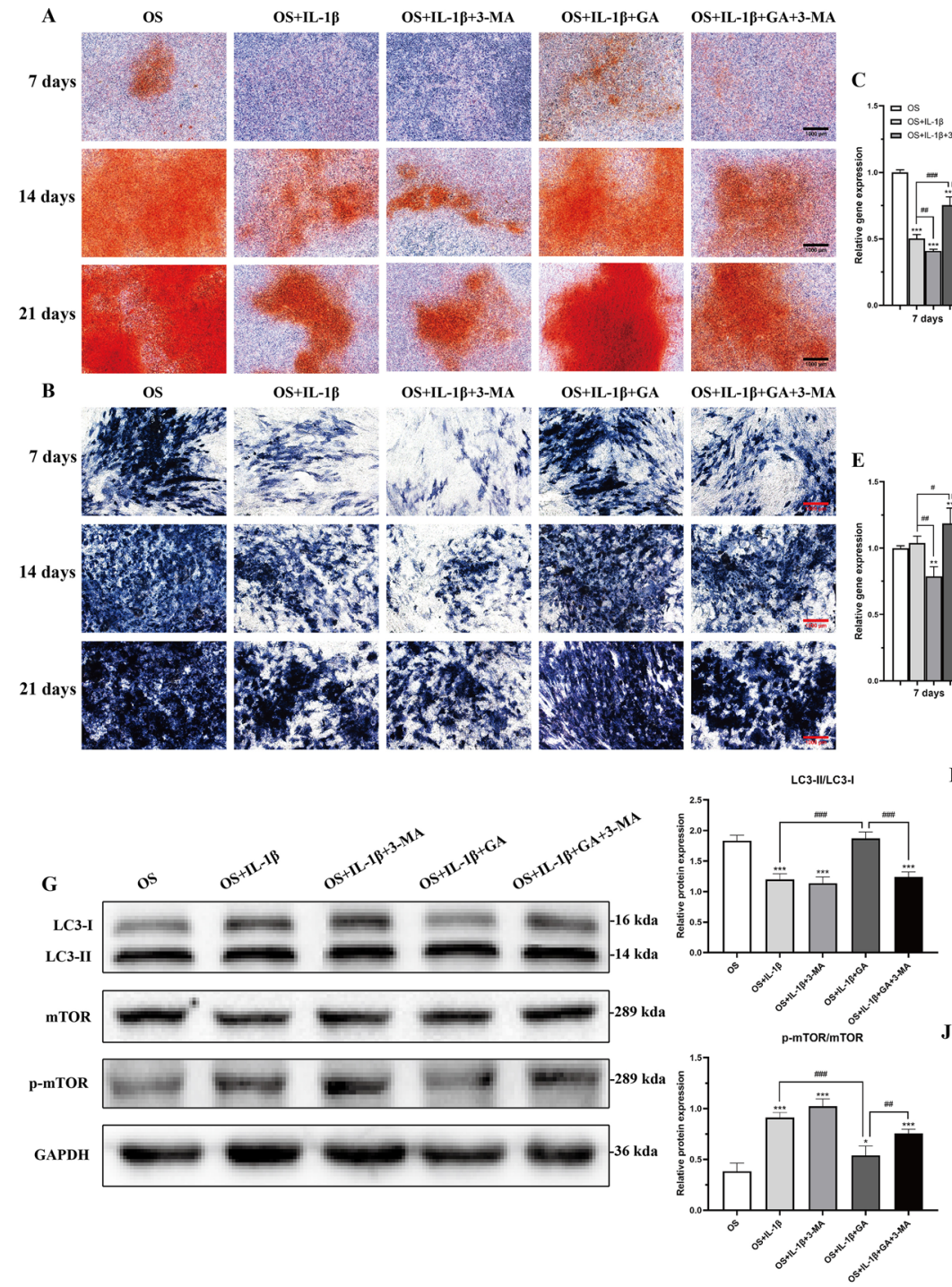
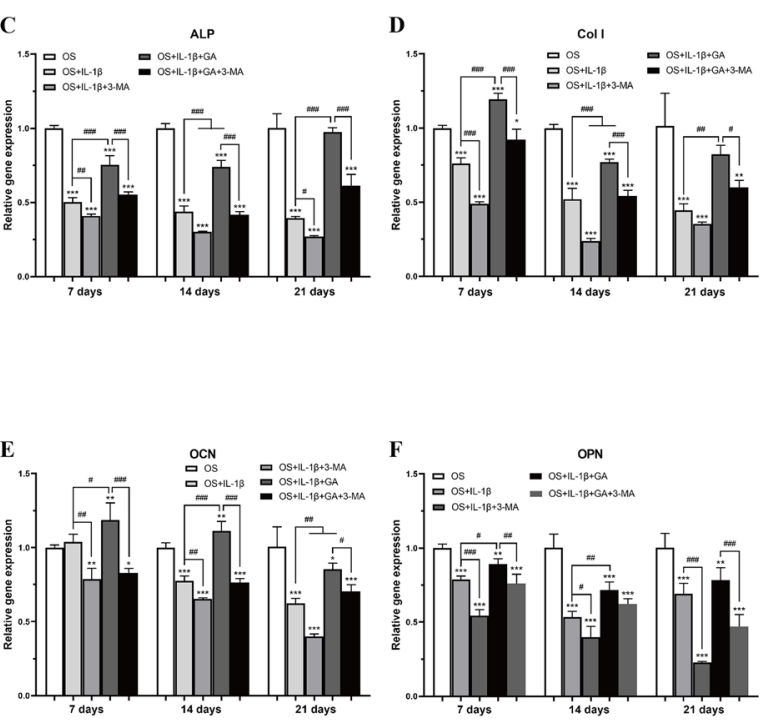

Figure 7. The effect of His6-GABARAP on IL-1 $\beta$-induced BMSCs osteogenic differentiation. Qualitative assessment of calcium deposition in BMSCs was stained by Alizarin Red (A) and ALP (B) staining after 7, 14 , and 21 days of osteogenic differentiation culture. (C-F, and $\mathbf{H}-\mathbf{K})$ qRT-PCR was used to analyze the gene expression levels of ALP, Col I, OCN, OPN, Atg5, Atg7, Beclin-1, and mTOR during osteogenic differentiation. (G) Western blot was used to analyze the protein expression of LC3, mTOR, and p-mTOR. OS (with osteogenic differentiation); OS + IL-1 $\beta$ (with osteogenic differentiation and $10 \mathrm{ng} / \mathrm{mL}$ IL-1 $\beta$ ); OS + IL-1 $\beta$ + 3-MA (with osteogenic differentiation, $10 \mathrm{ng} / \mathrm{mL}$ IL-1 $\beta$, and 3-MA); OS + IL- $1 \beta+\mathrm{GA}$ (with osteogenic differentiation, $10 \mathrm{ng} /$ $\mathrm{mL}$ IL-1 $\beta$, and $60 \mathrm{nM}$ His6-GABARAP); OS + IL-1 $\beta+\mathrm{GA}+3-\mathrm{MA}$ (with osteogenic differentiation, $10 \mathrm{ng} /$ mL IL-1 $\beta, 60 \mathrm{nM}$ His6-GABARAP, and 3-MA). Values are presented as means $\pm \mathrm{SD}, \mathrm{n}=3 .{ }^{*} p<0.05,{ }^{* *} p<0.01$,

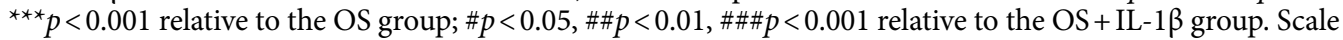
bar, $1000 \mu \mathrm{m}$.

Intracellular ROS generation is a major pathological driver of apoptosis in $\mathrm{BMSCs}^{47}$, which in turn plays a vital role in triggering inflammation ${ }^{48,49}$. Studies have demonstrated that autophagy plays a key role in suppressing ROS formation ${ }^{50}$; therefore, we examined the effect of GABARAP on ROS generation in BMSCs. GABARAP stimulation significantly decreased ROS levels in BMSCs and the effect was maintained when co-cultured with IL- $1 \beta$. In addition, His6-GABARAP treatment increased the expression of MnSOD and CuZnSOD, which are antioxidant factors in the superoxide dismutase pathways ${ }^{51}$.

Autophagy is a catabolic process wherein damaged proteins and organelles are phagocytosed and degraded to maintain energy levels and support organelle renewa ${ }^{52}$. Under stress conditions such as hypoxia and starvation, activation of autophagy can prevent apoptosis and maintain cellular homeostasis ${ }^{53-55}$. Indeed, we found that autophagic flux was significantly suppressed in IL-1 $\beta$-induced BMSCs and restored by His6-GABARAP treatment. The phosphoinositide 3-kinase (PI3K) blocker 3-MA, which is routinely used to inhibit autophagy ${ }^{56}$, markedly abrogated the effects of GABARAP on apoptosis, the inflammatory response, and ROS generation. 
Therefore, our findings suggest that GABARAP promotes BMSCs proliferation and reduces inflammationinduced apoptosis by activating autophagy; however, more studies are required to clarify the mechanism further.

The capacity to differentiate into several cell lines is a crucial characteristic of BMSCs, and it has been reported that the stimulation of intracellular ROS degradation by autophagy activation plays a vital role in regulating BMSCs osteogenic differentiation ${ }^{27}$. In this study, we observed that the pharmacological induction of GABARAP was closely associated with BMSCs osteogenesis and calcium deposition. Due to the importance of IL- $1 \beta$ in MSCs differentiation, multiple studies have demonstrated that IL-1 $\beta$ plays a crucial role in suppressing osteogenesis ${ }^{57}$ and upregulating metalloproteinase expression to degrade the extracellular matrix of bone tissue ${ }^{18}$. Conversely, studies have also suggested that IL- $1 \beta$ stimulation promotes calcium deposition and endochondral ossification $^{58,59}$; however, recent studies have shown that the effects of IL- $1 \beta$ on stem cells is dependent upon the target cell line and the IL- $1 \beta$ concentration ${ }^{36}$. Notably, in our study BMSCs displayed a lower osteoblast capacity when incubated with $10 \mathrm{ng} / \mathrm{mL}$ of IL-1 $\beta$, with His6-GABARAP treatment improving osteogenic differentiation in the presence of IL- $1 \beta$ via a mechanism closely related to the up-regulation of autophagy markers. As hypothesized, 3-MA stimulation effectively inhibited the effect of GABARAP, suggesting that GABARAP stimulates the osteogenic differentiation of IL- $1 \beta$-induced BMSCs by activating autophagy. This stimulatory effect of GABARAP may be due to the role of autophagy maintenance in ROS generation, since autophagy has been shown to be a good candidate for maintaining the redox homeostasis of BMSCs ${ }^{27}$. Moreover, elevated ROS levels in BMSCs have been reported to modulate osteogenesis, with ROS scavenging able to effectively restore their osteogenic capacity $^{60}$. However, the specific mechanisms during osteogenic differentiation, particularly in the presence of GABARAP, require further exploration. Taken together, we showed that GABARAP promotes osteogenic differentiation in IL- $1 \beta$-induced BMSCs, at least partly by activating the autophagy pathway.

In conclusion, GABARAP improves BMSCs proliferation and partially protects cell against IL-1 $\beta$-induced inflammation and intracellular ROS generation. In addition, our findings suggest that GABARAP promotes the osteogenic differentiation of BMSCs exposed to IL- $1 \beta$ and that autophagy activation is involved in its effect on BMSCs viability and osteogenic differentiation. Further studies will be conducted focusing on the mechanisms underlying the effects of GABARAP under more complicated inflammatory conditions. However, GABARAP may represent a novel therapeutic option for treating inflammation in OA and an effective method for promoting bone tissue regeneration.

\section{Data availability}

The data used to support the findings of this study are available from the corresponding author upon request.

Received: 16 March 2021; Accepted: 13 May 2021

Published online: 02 June 2021

\section{References}

1. Sakaguchi, Y., Sekiya, I., Yagishita, K. \& Muneta, T. Comparison of human stem cells derived from various mesenchymal tissues: Superiority of synovium as a cell source. Arthritis Rheumatol. 52, 2521-2529 (2005).

2. He, F., Chen, X. \& Pei, M. Reconstruction of an in vitro tissue-specific microenvironment to rejuvenate synovium-derived stem cells for cartilage tissue engineering. Tissue Eng. Part A 15, 3809-3821 (2009).

3. Bari, C. D., Dell'Accio, F., Tylzanowski, P. \& Luyten, F. P. Multipotent mesenchymal stem cells from adult human synovial membrane. Arthritis Rheumatol. 44, 1928-1942 (2001).

4. Codispoti, B., Marrelli, M., Paduano, F. \& Tatullo, M. NANOmetric BIO-banked MSC-derived exosome (NANOBIOME) as a Novel approach to regenerative medicine. J. Clin. Med. https://doi.org/10.3390/jcm7100357 (2018).

5. Chen, K., Man, C., Zhang, B., Hu, J. \& Zhu, S.-S. Effect of in vitro chondrogenic differentiation of autologous mesenchymal stem cells on cartilage and subchondral cancellous bone repair in osteoarthritis of temporomandibular joint. Int. J. Oral Maxillofac. Surg. 42, 240-248 (2013).

6. Gao, B. et al. Melatonin rescued interleukin lbeta-impaired chondrogenesis of human mesenchymal stem cells. Stem Cell Res. Ther. 9, 162. https://doi.org/10.1186/s13287-018-0892-3 (2018).

7. Deng, X., Jing, D., Liang, H., Zheng, D. \& Shao, Z. H(2)O(2) Damages the stemness of rat bone marrow-derived mesenchymal stem cells: developing a "Stemness Loss" model. Med. Sci. Monit. : Int. Med. J. Exp. Clin. Res. 25, 5613-5620. https://doi.org/10. $12659 / \mathrm{msm} .914011(2019)$.

8. Wang, X. et al. Hsp20-engineered mesenchymal stem cells are resistant to oxidative stress via enhanced activation of Akt and increased secretion of growth factors. Stem Cells 27(12), 3021-3031 (2009).

9. Huang, J. \& Chen, L. IL-1 $\beta$ inhibits osteogenesis of human bone marrow-derived mesenchymal stem cells by activating FoxD3/ microRNA-496 to repress Wnt signaling. Genesis 55(7), e23040 (2017).

10. Dzau, V. J., Hodgkinson, C. P., Gomez, J. A. \& Mirotsou, M. Genetic engineering of mesenchymal stem cells and its application in human disease therapy. Hum. Gene Therapy 21(11), 1513-1526 (2010).

11. Ru, L. B. \& Terkeltaub, R. Emerging regulators of the inflammatory process in osteoarthritis. Nat. Rev. Rheumatol. 11, 35 (2015).

12. Lee, Y. M., Fujikado, N., Manaka, H., Yasuda, H. \& Iwakura, Y. IL-1 plays an important role in the bone metabolism under physiological conditions. Int. Immunol. 22, 805-816 (2010).

13. Nogueira, A. V., de Molon, R. S., Nokhbehsaim, M., Deschner, J. \& Cirelli, J. A. Contribution of biomechanical forces to inflammation-induced bone resorption. J. Clin. Periodontol. 44, 31-41. https://doi.org/10.1111/jcpe.12636 (2017).

14. Zhang, S. et al. MSC exosomes alleviate temporomandibular joint osteoarthritis by attenuating inflammation and restoring matrix homeostasis. Biomaterials 200, 35-47. https://doi.org/10.1016/j.biomaterials.2019.02.006 (2019).

15. Yang, R. et al. Activation of the Wnt/ $\beta$-catenin pathway by an inflammatory microenvironment affects the myogenic differentiation capacity of human laryngeal mucosa mesenchymal stromal cells. Stem Cells Dev. 27, 771-782 (2018).

16. Yang, H. et al. Therapeutic effect of TSG-6 engineered iPSC-derived MSCs on experimental periodontitis in rats: a pilot study. PLoS ONE 9, e100285. https://doi.org/10.1371/journal.pone.0100285 (2014).

17. Liu, X. et al. Melatonin mediates protective effects on inflammatory response induced by interleukin-1 beta in human mesenchymal stem cells. J. Pineal Res. 55, 14-25. https://doi.org/10.1111/jpi.12045 (2013).

18. Pecchi, E. et al. A potential role of chondroitin sulfate on bone in osteoarthritis: inhibition of prostaglandin $\mathrm{E}(2)$ and matrix metalloproteinases synthesis in interleukin-1beta-stimulated osteoblasts. Osteoarthritis Cartil. 20, 127-135. https://doi.org/10.1016/j. joca.2011.12.002 (2012). 
19. Zhao, H. et al. Chemokine-like receptor 1 deficiency leads to lower bone mass in male mice. Cell. Mol. Life Sci. (CMLS) 76, 355-367. https://doi.org/10.1007/s00018-018-2944-3 (2019).

20. Liang, X. et al. Icariin promotes osteogenic differentiation of bone marrow stromal cells and prevents bone loss in OVX mice via activating autophagy. J. Cell. Biochem. 120, 13121-13132. https://doi.org/10.1002/jcb.28585 (2019).

21. Levine, B. \& Kroemer, G. Autophagy in the pathogenesis of disease. Cell 132, 27-42. https://doi.org/10.1016/j.cell.2007.12.018 (2008).

22. Pierrefite-Carle, V., Santucci-Darmanin, S., Breuil, V., Camuzard, O. \& Carle, G. F. Autophagy in bone: self-eating to stay in balance. Ageing Res. Rev. 24, 206-217. https://doi.org/10.1016/j.arr.2015.08.004 (2015).

23. Florencio-Silva, R. et al. Osteoporosis and autophagy: what is the relationship?. Rev. Assoc. Med. Bras. 1992(63), 173-179. https:// doi.org/10.1590/1806-9282.63.02.173 (2017).

24. Hou, J. et al. Autophagy prevents irradiation injury and maintains stemness through decreasing ROS generation in mesenchymal stem cells. Cell Death Dis. 4, e844. https://doi.org/10.1038/cddis.2013.338 (2013).

25. Hu, C., Zhao, L., Wu, D. \& Li, L. Modulating autophagy in mesenchymal stem cells effectively protects against hypoxia- or ischemiainduced injury. Stem Cell Res. Ther. 10, 120. https://doi.org/10.1186/s13287-019-1225-x (2019).

26. Chen, K., Yang, Y. H., Jiang, S. D. \& Jiang, L. S. Decreased activity of osteocyte autophagy with aging may contribute to the bone loss in senile population. Histochem. Cell Biol. 142, 285-295. https://doi.org/10.1007/s00418-014-1194-1 (2014).

27. Geng, W. et al. Substance P enhances BMSC osteogenic differentiation via autophagic activation. Mol. Med. Rep. 20, 664-670. https://doi.org/10.3892/mmr.2019.10257 (2019).

28. Koike, M. et al. Enrichment of GABARAP relative to LC3 in the axonal initial segments of neurons. PLoS ONE 8, e63568. https:// doi.org/10.1371/journal.pone.0063568 (2013).

29. Kabeya, Y. et al. LC3, GABARAP and GATE16 localize to autophagosomal membrane depending on form-II formation. J. Cell Sci. 117, 2805-2812 (2004).

30. Klebig, C. et al. Characterization of \{gamma\}-aminobutyric acid type A receptor-associated protein, a novel tumor suppressor, showing reduced expression in breast cancer. Can. Res. 65, 394-400 (2005).

31. Hu, H. et al. Gene expression profiles and bioinformatics analysis of human umbilical vein endothelial cells exposed to PM 25. Chemosphere 183, 589-598 (2017).

32. Park, S. H. et al. GABARBP down-regulates HIF-1 $\alpha$ expression through the VEGFR-2 and PI3K/mTOR/4E-BP1 pathways. Cell. Signal. 26, 1506-1513 (2014).

33. McGrath, J. C. \& Lilley, E. Implementing guidelines on reporting research using animals (ARRIVE etc.): new requirements for publication in BJP. Br. J. Pharmacol. 172, 3189-3193. https://doi.org/10.1111/bph.12955(2015) (2015).

34. McGrath, J. C., McLachlan, E. M. \& Zeller, R. Transparency in research involving animals: the basel declaration and new principles for reporting research in BJP manuscripts. Br. J. Pharmacol. 172, 2427-2432. https://doi.org/10.1111/bph.12956 (2015).

35. Yu, X. et al. LL-37 inhibits LPS-induced inflammation and stimulates the osteogenic differentiation of BMSCs via P2X7 receptor and MAPK signaling pathway. Exp. Cell Res. 372, 178-187. https://doi.org/10.1016/j.yexcr.2018.09.024 (2018).

36. Sullivan, C. B. et al. TNFalpha and IL-1beta influence the differentiation and migration of murine MSCs independently of the NF-kappaB pathway. Stem Cell Res. Ther. 5, 104. https://doi.org/10.1186/scrt492 (2014).

37. Thul, P. J. et al. A subcellular map of the human proteome. Science https://doi.org/10.1126/science.aal3321 (2017).

38. Wu, Z. et al. GABARAP promotes bone marrow mesenchymal stem cells-based the osteoarthritis cartilage regeneration through the inhibition of PI3K/AKT/mTOR signaling pathway. J. Cell. Physiol. 234, 21014-21026. https://doi.org/10.1002/jcp.28705 (2019).

39. Radin, E. L. \& Rose, R. M. Role of subchondral bone in the initiation and progression of cartilage damage. Clin. Orthop. Relat. Res. 213, 34-40 (1986).

40. Finnila, M. A. J. et al. Association between subchondral bone structure and osteoarthritis histopathological grade. J. Orthop. Res. Off. Publ. Orthop. Res. Soc. 35, 785-792. https://doi.org/10.1002/jor.23312 (2017).

41. Belluzzi, E. et al. Contribution of infrapatellar fat pad and synovial membrane to knee osteoarthritis pain. Biomed. Res. Int. 2019, 6390182. https://doi.org/10.1155/2019/6390182 (2019).

42. Li, L., Li, L., Zhang, Z. \& Jiang, Z. Hypoxia promotes bone marrow-derived mesenchymal stem cell proliferation through apelin/ APJ/autophagy pathway. Acta Biochim. Biophys. Sin. 47, 362-367. https://doi.org/10.1093/abbs/gmv014 (2015).

43. Chen, C. et al. Interleukin-1beta and tumor necrosis factor-alpha increase stiffness and impair contractile function of articular chondrocytes. Acta Biochim. Biophys. Sin. 47, 121-129. https://doi.org/10.1093/abbs/gmul16 (2015).

44. Yao, Z. Z., Hu, A. X. \& Liu, X. S. DUSP19 regulates IL-1beta-induced apoptosis and MMPs expression in rat chondrocytes through JAK2/STAT3 signaling pathway. Biomed. Pharmacother. 96, 1209-1215. https://doi.org/10.1016/j.biopha.2017.11.097 (2017).

45. Wang, X. et al. Tanshinone I inhibits IL-1beta-induced apoptosis, inflammation and extracellular matrix degradation in chondrocytes CHON-001 cells and attenuates murine osteoarthritis. Drug Des. Dev. Ther. 13, 3559-3568. https://doi.org/10.2147/dddt. S216596 (2019).

46. Bakker, A. D. et al. Tumor necrosis factor alpha and interleukin-1beta modulate calcium and nitric oxide signaling in mechanically stimulated osteocytes. Arthritis Rheumatol. 60, 3336-3345. https://doi.org/10.1002/art.24920 (2009).

47. Weinberg, E., Maymon, T. \& Weinreb, M. AGEs induce caspase-mediated apoptosis of rat BMSCs via TNFalpha production and oxidative stress. J. Mol. Endocrinol. 52, 67-76. https://doi.org/10.1530/jme-13-0229 (2014).

48. Musumeci, G. et al. Biomarkers of chondrocyte apoptosis and autophagy in osteoarthritis. Int. J. Mol. Sci. 16, 20560-20575. https:// doi.org/10.3390/ijms160920560 (2015).

49. Hwang, H. S. \& Kim, H. A. Chondrocyte apoptosis in the pathogenesis of osteoarthritis. Int. J. Mol. Sci. 16, 26035-26054. https:// doi.org/10.3390/ijms161125943 (2015).

50. Gao, Q. Oxidative stress and autophagy. Adv. Exp. Med. Biol. 1206, 179-198. https://doi.org/10.1007/978-981-15-0602-4_9 (2019).

51. Fischer, T. W., Kleszczynski, K., Hardkop, L. H., Kruse, N. \& Zillikens, D. Melatonin enhances antioxidative enzyme gene expression (CAT, GPx, SOD), prevents their UVR-induced depletion, and protects against the formation of DNA damage (8-hydroxy2'-deoxyguanosine) in ex vivo human skin. J. Pineal Res. 54, 303-312. https://doi.org/10.1111/jpi.12018 (2013).

52. Yin, H. et al. The therapeutic and pathogenic role of autophagy in autoimmune diseases. Front. Immunol. 9, 1512. https://doi.org/ 10.3389/fimmu.2018.01512 (2018).

53. Saito, S. \& Nakashima, A. Review: the role of autophagy in extravillous trophoblast function under hypoxia. Placenta 34(Suppl), S79-84. https://doi.org/10.1016/j.placenta.2012.11.026 (2013).

54. Kume, S., Thomas, M. C. \& Koya, D. Nutrient sensing, autophagy, and diabetic nephropathy. Diabetes 61, 23-29. https://doi.org/ $10.2337 / \mathrm{db} 11-0555$ (2012)

55. Poillet-Perez, L., Despouy, G., Delage-Mourroux, R. \& Boyer-Guittaut, M. Interplay between ROS and autophagy in cancer cells, from tumor initiation to cancer therapy. Redox Biol. 4, 184-192. https://doi.org/10.1016/j.redox.2014.12.003 (2015).

56. $\mathrm{Hu}$, J. et al. Globular adiponectin attenuated $\mathrm{H} 2 \mathrm{O} 2$-induced apoptosis in rat chondrocytes by inducing autophagy through the AMPK/mTOR pathway. Cell. Physiol. Biochem. Int. J. Exp. Cell. Physiol. Biochem. Pharmacol. 43, 367-382. https://doi.org/10.1159/ 000480416 (2017).

57. Wan, W. et al. Synergistic effect of matrix stiffness and inflammatory factors on osteogenic differentiation of MSC. Biophys. J. 117, 129-142. https://doi.org/10.1016/j.bpj.2019.05.019 (2019).

58. Lin, F. H., Chang, J. B., McGuire, M. H., Yee, J. A. \& Brigman, B. E. Biphasic effects of interleukin-1beta on osteoblast differentiation in vitro. J. Orthop. Res. Off. Publ. Orthop. Res. Soc. 28, 958-964. https://doi.org/10.1002/jor.21099 (2010). 
59. Lange, J. et al. Action of IL-1beta during fracture healing. J. Orthop. Res. Off. Publ. Orthop. Res. Soc. 28, 778-784. https://doi.org/ 10.1002/jor.21061 (2010).

60. Chen, H. et al. Role of SIRT1 and AMPK in mesenchymal stem cells differentiation. Ageing Res. Rev. 13, 55-64. https://doi.org/ 10.1016/j.arr.2013.12.002 (2014).

\section{Acknowledgements}

This work has been financially supported by Innovation Project of Guangxi Graduate Education (Grant No. YCSW2019117).

\section{Author contributions}

Z.W. and X.G. conceived and designed the study. X.G. analyzed data. Z.W. wrote this manuscript. All authors reviewed and approved the final manuscript.

\section{Competing interests}

The authors declare no competing interests.

\section{Additional information}

Supplementary Information The online version contains supplementary material available at https://doi.org/ 10.1038/s41598-021-90586-9.

Correspondence and requests for materials should be addressed to X.G.

Reprints and permissions information is available at www.nature.com/reprints.

Publisher's note Springer Nature remains neutral with regard to jurisdictional claims in published maps and institutional affiliations.

(c) (i) Open Access This article is licensed under a Creative Commons Attribution 4.0 International License, which permits use, sharing, adaptation, distribution and reproduction in any medium or format, as long as you give appropriate credit to the original author(s) and the source, provide a link to the Creative Commons licence, and indicate if changes were made. The images or other third party material in this article are included in the article's Creative Commons licence, unless indicated otherwise in a credit line to the material. If material is not included in the article's Creative Commons licence and your intended use is not permitted by statutory regulation or exceeds the permitted use, you will need to obtain permission directly from the copyright holder. To view a copy of this licence, visit http://creativecommons.org/licenses/by/4.0/.

(C) The Author(s) 2021 